TRANSACTIONS OF THE

AMERICAN MATHEMATICAL SOCIETY

Volume 359, Number 2, February 2007, Pages 909-922

S 0002-9947(06)03924-9

Article electronically published on September 12, 2006

\title{
COUNTING INTEGRAL LAMÉ EQUATIONS BY MEANS OF DESSINS D'ENFANTS
}

\author{
SANDER R. DAHMEN
}

\begin{abstract}
We obtain an explicit formula for the number of Lamé equations (modulo linear changes of variable) with index $n$ and projective monodromy group of order $2 N$, for given $n \in \mathbb{Z}$ and $N \in \mathbb{N}$. This is done by performing the combinatorics of the 'dessins d'enfants' associated to the Belyi covers which transform hypergeometric equations into Lamé equations by pull-back.
\end{abstract}

\section{INTRODUCTION}

The integral Lamé equation with parameters $B, e_{1}, e_{2}, e_{3} \in \mathbb{C}$ and $n \in \mathbb{Z}$ is the second order differential equation on the Riemann sphere $\mathbb{P}^{1}$ given by

$$
p(z) \frac{d^{2} y}{d z^{2}}+\frac{1}{2} p^{\prime}(z) \frac{d y}{d z}-(n(n+1) z+B) y=0,
$$

where $p(z):=4\left(z-e_{1}\right)\left(z-e_{2}\right)\left(z-e_{3}\right)$ is a polynomial with three different zeroes. The parameter $n$ is called the index. We are interested in Lamé equations with a basis of solutions which are algebraic (over $\mathbb{C}(z)$ ). Such solutions can in fact also occur for noninteger $n \in \mathbb{Q}$, but in this article we restrict to $n \in \mathbb{Z}$, hence the word integral in 'integral Lamé equation'.

It is known that if there exists a basis of algebraic solutions, then the (projective) monodromy group is dihedral; see for example [Bal, Thm. 1.5], BW, Cor. 3.4] or $\mathrm{vdW}$, Thm. 4.5.5]. If in (1.1) we make a linear change of the independent variable, i.e. substitute $z \mapsto a z+b(a, b \in \mathbb{C}, a \neq 0)$, we get a new Lamé equation with the same index $n$. These linear changes of variable induce a natural equivalence relation on the space of all Lamé equations; we will call two Lamé equations which are equivalent w.r.t. this equivalence relation linear equivalent. Now it is also known that for given $n \in \mathbb{Z}$ and $N \in \mathbb{N}$ there are only finitely many Lamé equations modulo linear equivalence with index $n$ and (projective) monodromy group dihedral of order $2 N$. This can be proven in different ways; see for example Chil, Remark 0.2 and Sec. 1] [BW, Thm. 4.6], vdW, Thms. 5.4.4, 6.7.9] or [Lit1, Thm. 4.1]. Throughout this article the number of Lamé equations modulo linear equivalence with index $n$ and projective monodromy group dihedral of order $2 N$ will be denoted by $L(n, N)$.

In [Lit1] it is described how the problem of calculating $L(n, N)$ can be translated in counting the number of dessins d'enfants compatible with prescribed ramification data. In the same article the combinatorics are performed for $n=1$, obtaining a result earlier obtained in Chi]. In Lit2] the combinatorics for $n=2$ are performed

Received by the editors June 25, 2004 and, in revised form, January 21, 2005.

2000 Mathematics Subject Classification. Primary 34L40, 34M15; Secondary 11F11, 14 H30.

Key words and phrases. Lamé equation, algebraic solution, monodromy, dessin d'enfants.

(C)2006 American Mathematical Society Reverts to public domain 28 years from publication 
(according to Chi] a formula for $L(2, N)$ was also obtained in Cop ). In this article we perform the combinatorics for general $n \in \mathbb{N}$; this is done in section 3 . Before that, we describe some preliminaries in section 2. Finally, in section 4 we briefly discuss some applications of the formula for $L(n, N)$. We obtain some counting formulas w.r.t. the full monodromy group and at the same time obtain information about zeroes of some modular forms.

\section{Preliminaries}

The integral Lamé equations with finite projective monodromy group can be obtained as pull-backs of hypergeometric equations. We will now briefly describe what we precisely mean by this and describe the pull-back maps that do the job. For a general introduction to the theory of ordinary linear differential equations we refer to vdW, Chapter 1] (short) or Poo (long).

Let $L y=0, L^{\prime} y=0$ be two second order Fuchsian differential equations on $\mathbb{P}^{1}$, and let $F: \mathbb{P}^{1} \rightarrow \mathbb{P}^{1}$ be a nonconstant meromorphic function. Then we say that $L y=0$ is a (weak) pull-back of $L^{\prime} y=0$ by $F$ if there exist independent solutions $f, g$ of $L y=0$ and $f^{\prime}, g^{\prime}$ of $L^{\prime} y=0$ such that $f / g=\left(f^{\prime} / g^{\prime}\right) \circ F$.

The hypergeometric equation with parameters $\lambda, \mu, \nu \in \mathbb{R}$ is the second order differential equation on $\mathbb{P}^{1}$ given by

$$
\frac{d^{2} y}{d z^{2}}+\left(\frac{1-\lambda^{2}}{4 z^{2}}+\frac{1-\mu^{2}}{4(z-1)^{2}}+\frac{\lambda^{2}+\mu^{2}-1-\nu^{2}}{4 z(z-1)}\right) y=0 .
$$

The local exponent differences at $0,1, \infty$ are given by the parameters $\lambda, \mu, \nu$, respectively (if $z \in \mathbb{P}^{1}-\{0,1, \infty\}$, then $z$ is a regular point of the hypergeometric equation). Schwarz gave the complete list of all the hypergeometric equations with finite projective monodromy group. Klein then gave a sublist of this list, called the basic Schwarz list, and proved that every second order Fuchsian differential equation on $\mathbb{P}^{1}$ has finite projective monodromy group if and only if it is a pull-back of a hypergeometric equation belonging to the basic Schwarz list. We remark that if one considers linear differential equations on general (compact) Riemann surfaces instead of just on the Riemann sphere, then analogous statements hold. For more information on this and for proofs of the statements given above, we refer to $\mathrm{BD}$ ] and $\mathrm{Bal}$.

It follows that the integral Lamé equation with finite projective monodromy group is also such a pull-back. There is one type of hypergeometric equation appearing on the basic Schwarz list which we shall be concerned with in this article from now on. Namely the hypergeometric equation with parameters $(\lambda, \mu, \nu)=$ $(1 / 2,1 / N, 1 / 2)$ with $N \in \mathbb{Z}_{>2}$, which has projective monodromy group dihedral of order $2 N$ and which we shall denote by $H_{N} y=0$. The main point of the counting method in Chi], Lit1, Lit2 and this article is the fact that every integral Lamé equation with finite projective monodromy group is a pull-back of a hypergeometric equation $H_{N} y=0$ by a covering $F: \mathbb{P}^{1} \rightarrow \mathbb{P}^{1}$ which is unramified outside $\{0,1, \infty\}$. In this case the Lamé equation has projective monodromy group dihedral of order $2 N^{\prime}$, with $N^{\prime} \mid N$; see [Bal], $\mathrm{BD}$. In [Chi] the ramification data of such coverings $F$ were computed.

Following [Lit1, we write the Lamé equation as in (1.1), but now with $p(z):=$ $4 z(z-1)(z-\lambda)$ and $\lambda \in \mathbb{C}-\{0,1\}$. According to Chi] (see also Lit1, Lit2) we have for every $n, N \in \mathbb{N}$ that a cover $F: \mathbb{P}^{1} \rightarrow \mathbb{P}^{1}$ transforms, by pull-back, the 
hypergeometric equation $H_{N} y=0$ into a Lamé equation with index $n$ and finite projective monodromy group (dihedral of order $2 N^{\prime}$, with $\left.N^{\prime} \mid N\right)$ if and only if the ramification data of $F$ are given by Tables 1, 2, 3, 4 or 5 below, with $(1, \infty, 0)$ in the first column replaced by $(0,1, \infty)$ or $(\infty, 1,0)$ (the tables are explained below).

TABle 1. Case Ia

\begin{tabular}{c|c|c|c|c|c} 
& 0 & 1 & $\lambda$ & $\infty$ & \\
\hline 1 & & & & & $+n N / 2$ points with multiplicity 2 \\
\hline$\infty$ & & & & & $+n$ points with multiplicity $N$ \\
\hline 0 & 1 & 1 & 1 & $2 \mathrm{n}+1$ & $+(n N-2 n-4) / 2$ points with multiplicity 2
\end{tabular}

TABle 2. Case Ib

\begin{tabular}{c|c|c|c|c|c} 
& 0 & 1 & $\lambda$ & $\infty$ & \\
\hline 1 & & & 1 & & $+(n N-1) / 2$ points with multiplicity 2 \\
\hline$\infty$ & & & & & $+n$ points with multiplicity $N$ \\
\hline 0 & 1 & 1 & & $2 \mathrm{n}+1$ & $+(n N-2 n-3) / 2$ points with multiplicity 2
\end{tabular}

TABle 3. Case Ic

\begin{tabular}{c|c|c|c|c|c} 
& 0 & 1 & $\lambda$ & $\infty$ & \\
\hline 1 & & 1 & 1 & & $+(n N-2) / 2$ points with multiplicity 2 \\
\hline$\infty$ & & & & & $+n$ points with multiplicity $N$ \\
\hline 0 & 1 & & & $2 \mathrm{n}+1$ & $+(n N-2 n-2) / 2$ points with multiplicity 2
\end{tabular}

TABle 4. Case Id

\begin{tabular}{c|c|c|c|c|c} 
& 0 & 1 & $\lambda$ & $\infty$ & \\
\hline 1 & 1 & 1 & 1 & & $+(n N-3) / 2$ points with multiplicity 2 \\
\hline$\infty$ & & & & & $+n$ points with multiplicity $N$ \\
\hline 0 & & & & $2 \mathrm{n}+1$ & $+(n N-2 n-1) / 2$ points with multiplicity 2
\end{tabular}

TABle 5. Case II

\begin{tabular}{c|c|c|c|c|c} 
& 0 & 1 & $\lambda$ & $\infty$ & \\
\hline 1 & & & & & $+n N / 2$ points with multiplicity 2 \\
\hline$\infty$ & & $\mathrm{N} / 2$ & $\mathrm{~N} / 2$ & & $+n-1$ points with multiplicity $N$ \\
\hline 0 & 1 & & & $2 \mathrm{n}+1$ & $+(n N-2 n-2) / 2$ points with multiplicity 2
\end{tabular}

The tables have the following meaning. All possible branching points of the cover $F: \mathbb{P}^{1} \rightarrow \mathbb{P}^{1}$, i.e. 0,1 and $\infty$, are contained in the first column. In the row where the entry in the first column equals $z \in\{0,1, \infty\}$, the number of points in the fiber $F^{-1}(z)$ together with their multiplicities are given as follows. In this row, an entry 
$m>0$ below $x \in\{0,1, \lambda, \infty\}$ means that $x \in F^{-1}(z)$ and that the multiplicity of $F$ at $x$ equals $m$, the number of points in the fiber $F^{-1}(z)$ not equal to $0,1, \lambda, \infty$ together with their multiplicities are given in the last column.

As mentioned in Chi], we do not need both the tables with in the first column $(1, \infty, 0)$ replaced by $(0,1, \infty)$ and the tables with in the first column $(1, \infty, 0)$ replaced by $(\infty, 1,0)$ to obtain all integral Lamé equations with finite monodromy by pull-back. The two sets of of tables are related by the transformation $z \mapsto 1 / z$, but the hypergeometric equation $H_{N} y=0$ is a pull-back of itself by this $1 / z$ transformation.

The covers $F$ above are examples of so-called Belyi functions. In general, if $X$ is a compact Riemann surface and $F: X \rightarrow \mathbb{P}^{1}$ is a cover unramified outside $\{0,1, \infty\}$, then $F$ is called a Belyi function and $(X, F)$ a Belyi pair. There is a remarkable theorem by Belyi, which states that a compact Riemann surface $X$ can be defined over $\overline{\mathbb{Q}}$ if and only if there exists a Belyi pair $(X, F)$ (in which case $F$ can also be defined over $\overline{\mathbb{Q}}$ ). Another remarkable fact about Belyi pairs is that they can be described in a purely combinatorial way as we shall (briefly) explain now. To a Belyi pair $(X, F)$ we can associate a bi-colored graph on the topological model of $X$ as follows. Let the (say) black vertices be given by $F^{-1}(0)$, the (say) white vertices by $F^{-1}(1)$ and the connecting edges by $F^{-1}((0,1))$ (where $(0,1)$ stands for the open interval $\{x \in \mathbb{R} \mid 0<x<1\}$ ). The graph we obtain is connected (any two different vertices are connected via a sequence of edges), bi-colored (every vertex is endowed with one of two colors, here black and white, and every edge connects a black vertex with a white vertex) and oriented (at every vertex a cyclic ordering of the edges leaving that vertex is given). Any connected, bi-colored, oriented graph is called a dessin d'enfants, or simply dessin. We consider two dessins d'enfants to be equivalent if there exists a graph isomorphism between them that preserves the bi-coloring and ordering. Two Belyi pairs $(X, F)$ and $\left(X^{\prime}, F^{\prime}\right)$ are considered equivalent if there exists an isomorphism $G: X \rightarrow X^{\prime}$ such that $F=F^{\prime} \circ G$. One easily checks that the construction above gives a well-defined map from the set of Belyi pairs modulo equivalence to the set of dessins d'enfants modulo equivalence. Remarkable is the fact that this map is actually a bijection, and is often called the Grothendieck correspondence. Now the ramification data of a Belyi pair $(X, F)$ correspond to the combinatorial data of the dessin associated to it. For example, let $x \in F^{-1}(\{0,1\})$. Then the multiplicity of $F$ at $x \in X$ equals the number of edges leaving the vertex $x$ of the corresponding dessin on $X$. Furthermore, $X-F^{-1}([0,1])$ consists of a finite union of simply connected open subsets $U_{1}, U_{2}, \ldots, U_{n}$, which could be called the faces of the dessin d'enfants. Every face contains exactly one inverse image of $\infty$, and the multiplicity of this point equals the number of edges that bound the face, where an edge counts only for $1 / 2$ if it is in the boundary of two different faces. In the rest of this article we shall be concerned only with dessins d'enfants on the Riemann sphere. For a further introduction to the theory of Belyi functions and dessins d'enfants we refer to [LZ, Chapter 2].

\section{The COMBINATORICS}

We will now perform the combinatorics of the dessins d'enfants associated to the pull-back maps described in the previous section, in order to obtain a formula for $L(n, N)$. 
Theorem 3.1. Let $n, N \in \mathbb{N}$. Then the number of dessins d'enfants compatible with the Tables 10 囵 equals $n(n+1)(N-1)(N-2) / 12+2 / 3 \varepsilon(n, N)$, where

$$
\varepsilon(n, N):= \begin{cases}1 & \text { if } 3 \mid N \text { and } n \equiv 1 \quad(\bmod 3), \\ 0 & \text { otherwise. }\end{cases}
$$

Proof. Let $n, N \in \mathbb{N}$ and suppose $n>1$. First consider Case I; it will not be necessary to consider the four cases Ia, Ib, Ic, Id separately. In all four cases there are $n$ points above $\infty$, all with multiplicity $N$. So the associated dessins consist of $n$ cells, all with valency $N$. Furthermore, there are $n-1$ cycles, which have beginning and end at the point $p:=\infty$, and 3 lines emanating from $p$. There are no further intersections. In this article, whenever we draw dessins d'enfants, we will not draw the vertices.

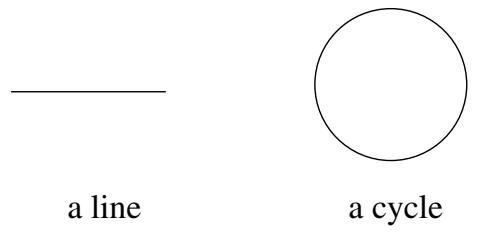

Figure 1. A line and a cycle, not taken into account the valencies

We define the valency of a line as the number of edges of that line and the valency of a cycle as half the number of edges of that cycle. With these definitions we define the valency of a cell as the sum of the valencies of the lines it contains and the cycles that bound it. We note that the valency of a cell equals the multiplicity of the inverse image of $\infty$ lying in the cell. We see that the boundary of every cell contains at least one cycle (since we assumed $n>1$ ). A cell with exactly one cycle in its boundary will be called a simple cell. Since all the cells have the same valency and there are only three lines, there can be no more than three simple cells. There is of course a minimum of two simple cells. These two cases, that of two simple cells (Case A) and that of three simple cells (Case B), will be considered separately.

We first consider Case A. If we do not take into account the 3 lines and the valencies of the cycles, it is easy to see that there is only one possibility for the dessin. It is of the form as given in Figure 2,

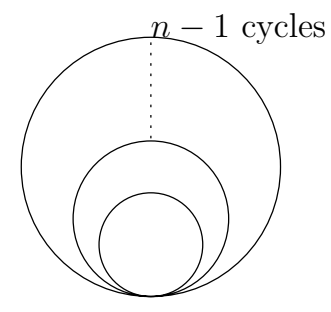

Figure 2. Case A

This can also be drawn in a more symmetric way as given in Figure 3 ,

We will now take into account the three lines (but still do not take into account the valencies). Each of the two simple cells must contain at least one line (since 
$n$ odd:

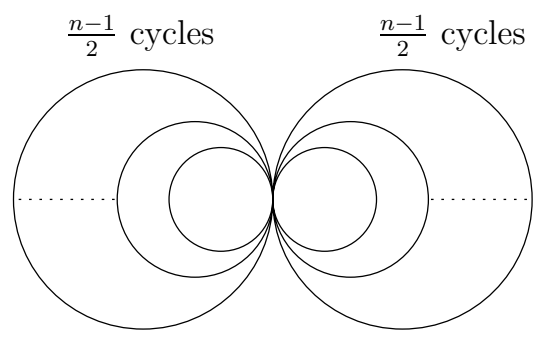

$n$ even:

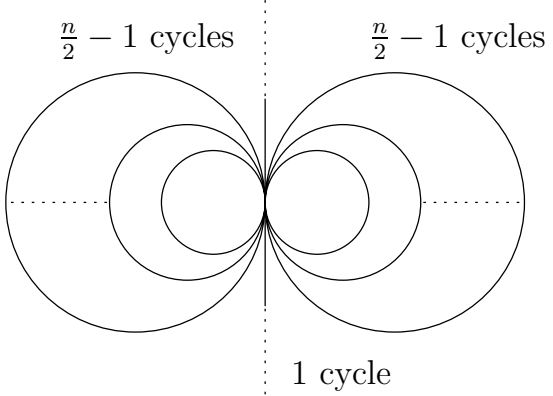

Figure 3. Case A

every cell has the same valency). If each of the three lines is contained in a simple cell, there is (because of rotational symmetry) only one possibility. We call this Case AI. The dessin is of the form as given in Figure 4.

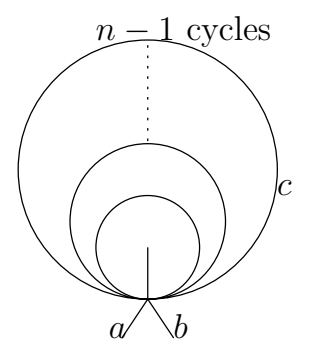

Figure 4. Case AI

If there is a line which is not contained in a simple cell, there are $n-2$ possible cells that can contain it. For each of these $n-2$ cells the line can lie on two different sides, but if we take into account the rotational symmetry, we see that the number of possibilities is reduced by a factor two. So in this case we arrive at exactly $n-2$ different kinds of dessins. We call this Case AII. The dessins are of the form as given in Figure 5, where the line which is not contained in a simple cell can always be drawn on the left side.

We will now take into account the valencies of the cycles and lines. Let $a, b, c \in \mathbb{N}$ denote the valencies as given in Figures 4 and 5 . In both cases we have $a+b+c=N$ (and $a, b, c \geq 1$ ). Furthermore, it is easy to see (by induction) that for every such triple $(a, b, c)$ there is exactly one possible dessin (for a fixed shape of the dessin). Now there are $(N-1)(N-2) / 2$ possible triples $(a, b, c)$. We conclude that there are exactly $(N-1)(N-2) / 2$ possible dessins of Type AI and there are exactly $(n-2)(N-1)(N-2) / 2$ possible dessins of Type AII. So there are exactly $(n-1)(N-1)(N-2) / 2$ possible dessins of Type A.

Now consider Case B. If we do not take into account the valencies of the cells and lines, one easily obtains (by induction) that the dessins are of the form as given in Figure 6. 


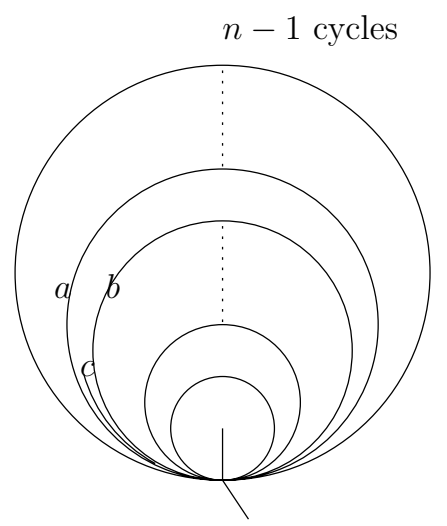

Figure 5. Case AII

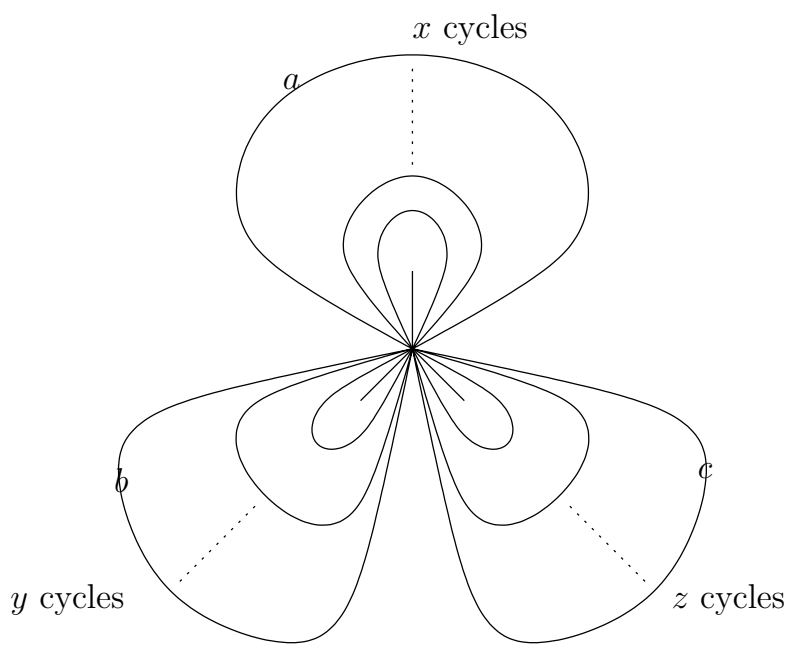

Figure 6. Case B

We first consider these dessins without taking into account rotational symmetry. Since $x+y+z=n-1$ (and $x, y, z \geq 1$ ) there are $(n-2)(n-3) / 2$ possibilities for the triple $(x, y, z)$. Note that for $n=2,3$ we also arrive at the correct answer, namely zero. We will now take into account the valencies of the cycles and lines. Let $a, b, c \in \mathbb{N}$ denote the valencies as given in Figure 6. We have $a+b+c=N$ (and $a, b, c \geq 1$ ). Furthermore, it is easy to see (by induction) that for every such triple $(a, b, c)$ there is exactly one possible dessin (for fixed $x, y, z$ and not yet taking into account the rotational symmetry). Now there are $(N-1)(N-2) / 2$ possible triples $(a, b, c)$. We conclude that there are $(n-2)(n-3)(N-1)(N-2) / 4$ possible dessins of Type B, not taking into account the rotational symmetry.

If we now do take into account the rotational symmetry, we see that we have counted every dessin three times, except when the dessin has a threefold rotational symmetry. There is exactly one dessin with a threefold rotational symmetry if and only if $N$ and the number of cycles are divisible by 3 , i.e. $3 \mid N$ and $n \equiv 1(\bmod 3)$, 
otherwise there are no such dessins. We conclude that there are exactly

$$
\frac{(n-2)(n-3)(N-1)(N-2)}{12}+\frac{2}{3} \varepsilon(n, N)
$$

possible dessins of Type B.

So the total number of dessins of Type I equals $(n-1)(N-1)(N-2) / 2+$ $(n-2)(n-3)(N-1)(N-2) / 12+2 / 3 \varepsilon(n, N)=n(n+1)(N-1)(N-2) / 12+$ $2 / 3 \varepsilon(n, N)$.

It remains to be proven that there are no dessins of Type II. In Case II there are $n+1$ points above $\infty, n-1$ with multiplicity $N$ and two with multiplicity $N / 2$. So the associated dessins consist of $n+1$ cells, $n-1$ with multiplicity $N$ and two with multiplicity $N / 2$. Furthermore, there are $n$ cycles and 1 line, which emanate from $p=\infty$. There are no further intersections. From the fact that there are two cells with valency strictly smaller than $N$, all other cells have valency $N$ and there is one line, we conclude that (again) there are either 2 simple cells (Case C) or 3 simple cells (Case D).

First consider the case of two simple cells. We distinguish the following two cases. Case CI: the line is contained in a simple cell. Case CII: the line is not contained in a simple cell. See Figure 7
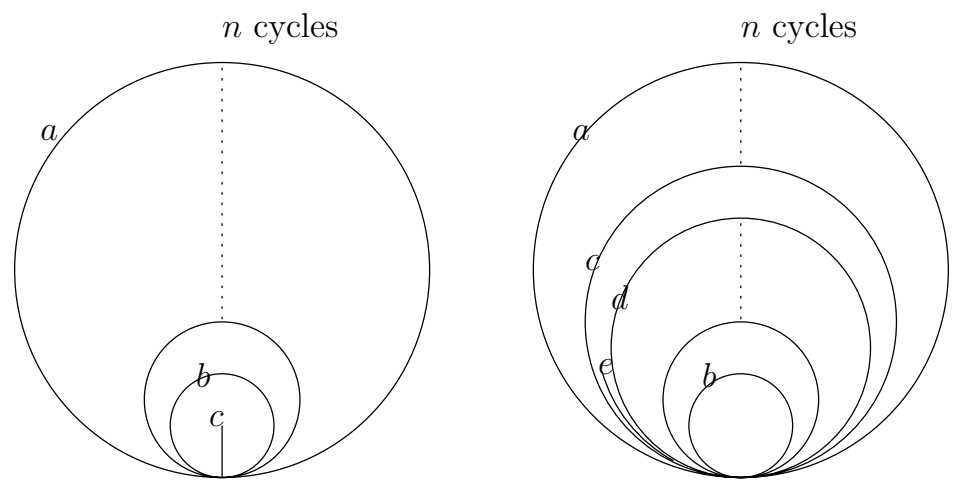

Figure 7. Left: Case CI; right: Case CII

Let $a, b, c \in \mathbb{N}$ denote the valencies as given in the left part of Figure 7 We must have $a=N / 2$, and by induction we obtain that all the nonsimple cells have valency $N$ and that $b=N / 2$. So $b+c>N / 2$, but this means that there is only one cell with valency $N / 2$. We conclude that there are no dessins of Type CI.

Let $a, b, c, d, e \in \mathbb{N}$ denote the valencies as given in the right part of Figure 7 We must have $a=b=N / 2$. Since all nonsimple cells must have valency $N$, we obtain by induction that $c=d=N / 2$. So $c+d+e>N$, but this means that there is a cell with valency larger that $N$. We conclude that there are no dessins of Type CII.

Now consider the case of three simple cells. The line must lie in a simple cell and the dessins are of the form as given in Figure 8

Let $a, b, c, d, e \in \mathbb{N}$ denote the valencies as given in Figure 8 . We must have $a=b=N / 2$. Since all nonsimple cells must have valency $N$ we obtain by induction that $c=d=N / 2$. So $c+d+e>N$, but this means that there is a cell with valency larger than $N$. We conclude that there are also no dessins of Type D, so there are 


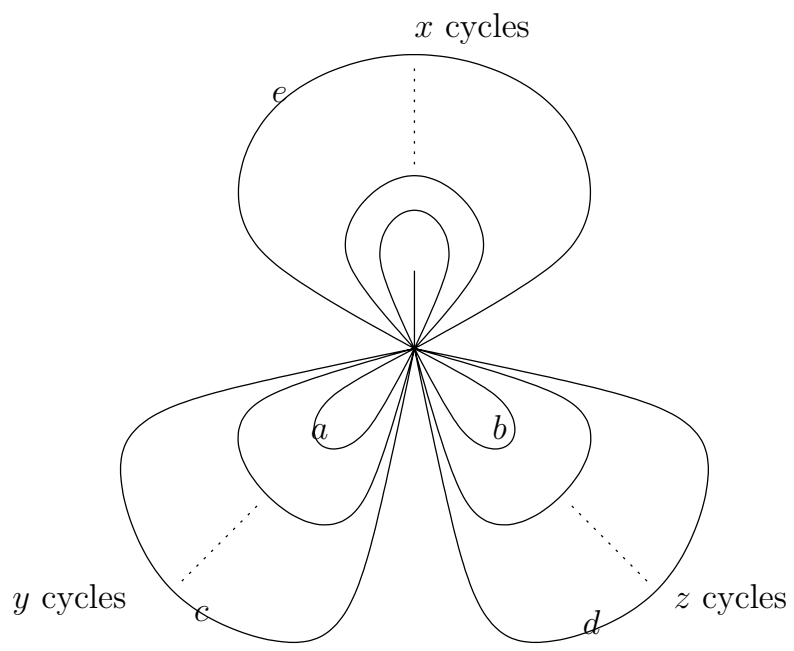

Figure 8. Case D

no dessins of Type II. We note that for $n=1$ the proof that there are no dessins of Type II also applies (but only Case CI has to be considered, and in that case there is only one cycle).

We have proven our theorem for $n>1$. For $n=1$ the combinatorics was done in Lit1] (and for $n=1$ the result was already obtained in Chi by other means), but the combinatorics can be simplified significantly as follows.

As noted before there are no dessins corresponding to Type II. So let us consider Case I. There is only one point above $\infty$, which has multiplicity $N$. So the associated dessins consist of one cell with valency $N$. Furthermore there are no cycles and three lines, which come together at the point $p=\infty$. The dessin is of the form as given in Figure 9 .

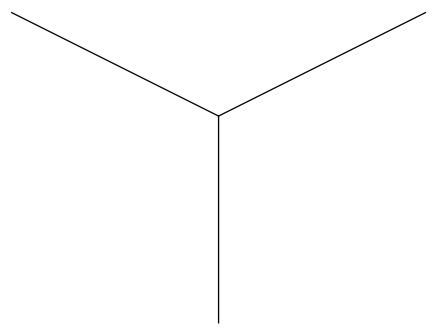

FiguRE 9. $n=1$

Let $a, b, c$ denote the valencies of the three lines. If we do not take into account the rotational symmetry, then the number of dessins equals the number of triples $(a, b, c)$ with $a+b+c=N$ (and $a, b, c \geq 1)$. There are $(N-1)(N-2) / 2$ such triples.

If we now take into account the rotational symmetry, we see that we have counted every dessin three times, except when the dessin has a threefold rotational symmetry. There is exactly one dessin with a threefold rotational symmetry if and only if 
$3 \mid N$, otherwise there are no such dessins. We conclude that there are exactly

$$
\frac{1}{3}\left(\frac{(N-1)(N-2)}{2}+2 \varepsilon(1, N)\right)=\frac{(N-1)(N-2)}{6}+\frac{2}{3} \varepsilon(1, N)
$$

possible dessins when $n=1$. This finishes the proof.

Now Theorem 3.1 gives rise to the following main result.

Theorem 3.2. Let $n \in \mathbb{Z}$ and $N \in \mathbb{N}$. Then

$$
\sum_{d \mid N} L(n, d)=\frac{n(n+1)}{12}(N-1)(N-2)+\frac{2}{3} \varepsilon(n, N),
$$

where $\varepsilon(n, N)$ is as in Theorem 3.1 .

Proof. This proof is essentially the same as the proof of Theorem 2.15 in Chi or the proof of Proposition 3.1 in Lit1. We summarize the main points. Let $N^{\prime} \in$ $\mathbb{N}, N^{\prime} \neq 2$. Then a Lamé equation with index $n \in \mathbb{N}$ and projective monodromy group dihedral of order $2 N^{\prime}$ is the pull-back of a hypergeometric equation $H_{N^{\prime}} y=0$ and the pull-back map is unique (up to homography); see Bal. Here and in the rest of this proof we use the convention that we restrict to the covers in Tables 15 with $(1, \infty, 0)$ in the first column replaced by $(0,1, \infty)$ (and do not take into consideration the covers with $(1, \infty, 0)$ replaced by $(\infty, 1,0)$; recall that these two sets of tables are related by the transformation $z \mapsto 1 / z)$. Furthermore, the hypergeometric equation $H_{N^{\prime}} y=0$ is a pull-back of the hypergeometric equation $H_{N} y=0$ if and only if $N^{\prime} \mid N$. In this case the pull-back map is also unique up to homography and composition with $z \mapsto 1 / z$; see Bal, proof of Thm. 2.15] or Lit1, Prop. 3.1]. For given $N, n$ the number of covers modulo homography that pull-back the hypergeometric equation $H_{N} y=0$ into a Lamé equation with index $n$ and finite projective monodromy group equals the number of dessins compatible with Tables 1.5. which is given by Theorem 3.1. Note that for $N=2$ this number is zero. The theorem readily follows.

Let $N \in \mathbb{N}$. We denote by $\phi(N)$ Euler's totient function, i.e.

$$
\phi(N):=|\{k \in \mathbb{Z} \mid 0 \leq k<N, \operatorname{gcd}(k, N)=1\}|,
$$

and by $\Psi(N)$ its two-dimensional analogue, i.e.

$$
\Psi(N):=\left|\left\{\left(k_{1}, k_{2}\right) \in \mathbb{Z}^{2} \mid 0 \leq k_{1}, k_{2}<N, \operatorname{gcd}\left(k_{1}, k_{2}, N\right)=1\right\}\right| .
$$

From the theorem above, together with the well-known results

$$
\sum_{d \mid N} \phi(d)=N \text { and } \sum_{d \mid N} \Psi(d)=N^{2},
$$

we easily obtain the following.

Corollary 3.3. Let $n \in \mathbb{Z}$ and $N \in \mathbb{N}$. Then

$$
L(n, N)= \begin{cases}0 & \text { if } N=1, \\ \frac{n(n+1)}{12}(\Psi(N)-3 \phi(N))+\frac{2}{3} \epsilon(n, N) & \text { otherwise, }\end{cases}
$$

where

$$
\epsilon(n, N):= \begin{cases}1 & \text { if } N=3 \text { and } n \equiv 1 \quad(\bmod 3), \\ 0 & \text { otherwise. }\end{cases}
$$


We end this section with a remark about how to get rid of the $\epsilon(n, N)$ in Corollary 3.3 and the $\varepsilon(n, N)$ in Theorems 3.1 and 3.2. To make calculations easy we write the Lamé equation as in (1.1), but now with $p(z):=4 z^{3}-g_{2} z-g_{3}$, where $g_{2}, g_{3} \in \mathbb{C}$ and $g_{2}^{3}-27 g_{3}^{2} \neq 0$. For the moment fix the index $n \in \mathbb{N}$. Then a Lamé equation is given by the parameters $\left(B, g_{2}, g_{3}\right)$, where $B, g_{2}, g_{3} \in \mathbb{C}$ and $g_{2}^{3}-27 g_{3}^{2} \neq 0$. If in our Lamé equation we substitute $z \mapsto z / \lambda(\lambda \in \mathbb{C}-\{0\})$, then we get a new Lamé equation, but with the parameters $\left(B, g_{2}, g_{3}\right)$ replaced by $\left(\lambda B, \lambda^{2} g_{2}, \lambda^{3} g_{3}\right)$. This defines an action of $\mathbb{C}-\{0\}$ on the parameter space $\left\{\left(B, g_{2}, g_{3}\right) \in \mathbb{C}^{3} \mid g_{2}^{3}-27 g_{3}^{2} \neq 0\right\}$ of Lamé equations (with fixed index $n$ ). Of course the orbits are in bijective correspondence with the classes of Lamé equations modulo linear equivalence. Let us consider the stabilizer of a triple $\left(B, g_{2}, g_{3}\right)$. If $B \neq 0$, then the stabilizer is trivial. If $B=0$, but $g_{2} g_{3} \neq 0$, then the stabilizer is also trivial. The only two equivalence classes of Lamé equations where the stabilizer is not trivial are given by $B=g_{2}=0$ (in which case $g_{3} \neq 0$ ) and $B=g_{3}=0$ (in which case $\left.g_{2} \neq 0\right)$. In the first case the stabilizer has order 3 and in the second case it has order 2. Since in fact we count Lamé equations modulo the action given above, it is natural to assign a multiplicity to a (equivalence class of a) Lamé equation, equal to 1 /(the order of the corresponding stabilizer). This amounts to assigning a multiplicity of $1 / 3$ to the equivalence class of Lamé equations corresponding to $B=g_{2}=0$, a multiplicity of $1 / 2$ to the equivalence class of Lamé equations corresponding to $B=g_{3}=0$ and a multiplicity of 1 to all other equivalence classes of Lamé equations. The integral Lamé equations with finite projective monodromy group and multiplicity not equal to one are characterized by the following result.

Theorem 3.4. There does not exist a Lamé equation with index $n \in \mathbb{N}, B=g_{3}=0$ and finite projective monodromy group. There exists a Lamé equation with index $n \in \mathbb{N}, B=g_{2}=0$ and finite projective monodromy group dihedral of order $2 N$ if and only if $N=3$ and $n \equiv 1 \bmod 3$.

Proof. The theorem follows from vdW, Thm. 5.5.4 and Thm. 5.5.8]. Note however, that in loc. cit. the full monodromy group is considered instead of the projective monodromy group, and that the Lamé equations with $N=3$ are obtained as pull-backs of hypergeometric equations with local exponent differences at the singular points equal to $1 / 2,1 / 2+k$ and $1 / 3$, with $k \in \mathbb{Z}$.

From this result it follows immediately that if we count with the multiplicities as described above, then the $\epsilon(n, N)$ in Corollary 3.3 and the $\varepsilon(n, N)$ in Theorem 3.2 disappear. If we also count dessins with the corresponding multiplicities, then of course the $\varepsilon(n, N)$ in Theorem 3.1 also disappears. The dessins of the covers which transform, by pull-back, hypergeometric equations into Lamé equations with $B=g_{2}=0$ are given in Figure 6] (for $n>1$ and $x=y=z=(n-1) / 3$ and $a=b=c=N / 3$ ) and Figure 9 (for $n=1$ and the valencies of the lines all equal to $N / 3$ ). These dessins possess a threefold rotational symmetry, all other dessins possess no rotational symmetry.

\section{Applichtions}

We have counted the number of integral Lamé equations with given index and given finite projective monodromy group, but what about counts for the full monodromy group? If an integral Lamé equation has finite full monodromy group $M$, 
then (with respect to an appropriate basis) it is of the form

$$
M=\left\langle\left(\begin{array}{cc}
\zeta & 0 \\
0 & \zeta^{-1}
\end{array}\right),\left(\begin{array}{ll}
0 & 1 \\
1 & 0
\end{array}\right)\right\rangle
$$

where $\zeta$ is a primitive $N$-th root of unity for a certain $N \geq 3$; see [BW, Sec. 3 ] or vdW, Sec. 4.5]. The projective monodromy group of this Lamé equation is then isomorphic to $M$ modulo scalar matrices, which we shall denote by $P M$. The scalar matrices contained in $M$ are $\pm I$ if $N$ is even and $I$ if $N$ is odd. So we see that $P M$ is isomorphic to $D_{N / 2}$ if $N$ is even and $P M$ is isomorphic to $D_{N}$ if $N$ is odd (here and in the rest of this section $D_{N}$ stands for the dihedral group of order $2 N$ ). Write $F L(n, N)$ for the number of Lamé equations (modulo linear equivalence) with index $n$ and full monodromy group isomorphic to $D_{N}$. We have

$$
L(n, N)= \begin{cases}F L(n, N)+F L(n, 2 N) & \text { if } N \text { is odd } \\ F L(n, 2 N) & \text { if } N \text { is even }\end{cases}
$$

In [BW, Sec. 6] a method is presented to establish for which $N \geq 3$ there exists a Lamé equation with index $n=1$ and full monodromy group isomorphic to $D_{N}$. In fact, this method provides us with an upper bound for $F L(1, N)$. Broadly speaking, the method consists of first pulling back the Lamé equation to a differential equation on an elliptic curve (the so-called elliptic function form of the Lamé equation). Then explicit solutions of this equation are given and used to derive an explicit condition for the existence of a Lamé equation with full monodromy group isomorphic to $D_{N}$. The result is the following. Let $N \geq 3$. Then there exists a Lamé equation with index $n=1$ and full monodromy group isomorphic to $D_{N}$ if and only if

$$
\zeta\left(\frac{k_{1} \omega_{1}+k_{2} \omega_{2}}{N}, \omega_{1}, \omega_{2}\right)-\frac{k_{1} \eta_{1}+k_{2} \eta_{2}}{N}=0
$$

for some $k_{1}, k_{2} \in \mathbb{Z}$ with $\operatorname{gcd}\left(k_{1}, k_{2}, N\right)=1$. Here $\omega_{1}, \omega_{2} \in \mathbb{C}$ denote generators for the lattice $\Lambda \subset \mathbb{C}$ which is associated to the elliptic curve given by the equation $y^{2}=p(z)$. The function $u \mapsto \zeta\left(u, \omega_{1}, \omega_{2}\right)$ is the classical quasi-periodic Weierstraß zeta function relative to the lattice with generators $\omega_{1}, \omega_{2}$ (which is $\Lambda$ ) and $\eta_{1}, \eta_{2}$ are the quasi-periods defined by $\eta_{i}:=\zeta\left(u+\omega_{i}, \omega_{1}, \omega_{2}\right)-\zeta\left(u, \omega_{1}, \omega_{2}\right), i=1,2$. For more information about these classical objects we refer to WW, Chapter XX] (note however that there the symbols $\omega_{1}, \omega_{2}$ denote half-periods of $\Lambda$, instead of whole periods of $\Lambda$ as above). The functions given by

$$
Z_{N}\left(\omega_{1}, \omega_{2}, k_{1}, k_{2}\right):=\zeta\left(\frac{k_{1} \omega_{1}+k_{2} \omega_{2}}{N}, \omega_{1}, \omega_{2}\right)-\frac{k_{1} \eta_{1}+k_{2} \eta_{2}}{N}
$$

were already studied by Hecke in [Hec, Sec. 6]. The functions $Z_{N}\left(\tau ; k_{1}, k_{2}\right):=$ $Z_{N}\left(\tau, 1, k_{1}, k_{2}\right)$ are modular forms of weight one (w.r.t. $\left.\Gamma(N)\right)$ and now, broadly speaking, the equivalence classes of Lamé equations with index $n=1$ and full monodromy group isomorphic to $D_{N}$ are in correspondence with the zeroes of the modular forms $Z_{N}\left(\tau ; k_{1}, k_{2}\right)$, with $\operatorname{gcd}\left(k_{1}, k_{2}, N\right)=1$.

From the analysis given in the proof of [BW, Thm. 6.1] it follows that for $N \geq 3$ we have

$$
F L(1, N) \leq \frac{1}{2}\left(\frac{\Psi(N)}{12}-\phi\left(\frac{N}{2}\right)\right)+\frac{2}{3} \delta_{N, 3},
$$


where $\delta_{N, 3}$ stands for the Kronecker delta, i.e. it equals 1 if $N=3$ and equals 0 otherwise. And as before, $\phi$ and $\Psi$ denote Euler's totient function and its twodimensional analogue respectively, but with the extra convention that $\phi(N / 2)=0$ if $N / 2 \notin \mathbb{N}$. Moreover, the proof tells us that for $N \geq 3$ equality in (4.2) holds if and only if the modular forms $Z_{N}\left(\tau ; k_{1}, k_{2}\right)$, where $k_{1}, k_{2} \in \mathbb{Z}$ and $\operatorname{gcd}\left(k_{1}, k_{2}, N\right)=1$, have no multiple zeroes in the upper half plane. This last statement seems like something which is not easily proven. But luckily we have an exact counting formula w.r.t. the projective monodromy group. From inequality (4.2), equation (4.1) and the counting formula for $n=1$ in Corollary 3.3 , we readily obtain that equality holds in (4.2). As a bonus we obtain nontrivial information about classical modular forms as stated in the following theorem.

Theorem 4.1. Let $N \in \mathbb{Z}_{\geq 3}$ and $k_{1}, k_{2} \in \mathbb{Z}$ with $\operatorname{gcd}\left(k_{1}, k_{2}, N\right)=1$. Then every zero $\tau \in \mathfrak{H}:=\{z \in \mathbb{C} \mid \operatorname{Im}(z)>0\}$ of the modular form $Z_{N}\left(\tau ; k_{1}, k_{2}\right)$ is simple.

The counting method in BW, Sec. 6] can also be used (as main ingredient) to obtain upper bounds for $F L(n, N)$ when $n=2,3$. The result is that for $N \in \mathbb{Z}_{\geq 3}$ we have

$$
\begin{aligned}
& F L(2, N) \leq \frac{1}{2}\left(\frac{\Psi(N)}{4}-\left(\phi(N)+\phi\left(\frac{N}{2}\right)\right)\right), \\
& F L(3, N) \leq \frac{1}{2}\left(\frac{\Psi(N)}{2}-\left(\phi(N)+4 \phi\left(\frac{N}{2}\right)\right)\right) .
\end{aligned}
$$

Again, from these inequalities together with (4.1) and the counting formulas w.r.t. the projective monodromy group for $n=2,3$ in Corollary 3.3, we obtain that equality holds in the inequalities above. We summarize this result together with that for $n=1$ as follows.

Theorem 4.2. For every $N \in \mathbb{Z}_{\geq 3}$ we have

$$
\begin{aligned}
& F L(1, N)=\frac{1}{2}\left(\frac{\Psi(N)}{12}-\phi\left(\frac{N}{2}\right)\right)+\frac{2}{3} \delta_{N, 3}, \\
& F L(2, N)=\frac{1}{2}\left(\frac{\Psi(N)}{4}-\left(\phi(N)+\phi\left(\frac{N}{2}\right)\right)\right), \\
& F L(3, N)=\frac{1}{2}\left(\frac{\Psi(N)}{2}-\left(\phi(N)+4 \phi\left(\frac{N}{2}\right)\right)\right),
\end{aligned}
$$

where $\delta_{N, 3}$ equals 1 if $N=3$ and equals zero otherwise.

Again as a bonus we also obtain the result that some families of modular forms have no multiple zeroes in the upper half plane, but these modular forms are more complicated to describe than those in Theorem 4.1 (for example, the modular forms associated to $n=2,3$ are of weight 3,6 , respectively). More details and proofs will be described in a forthcoming article.

\section{ACKNOWLEDGMENTS}

The author would like to thank the referee for the useful comments and suggestions that found their way into this article, and also Frits Beukers for his support and for pointing out the articles [Lit1] and [Lit2]. 


\section{REFERENCES}

[Bal] F. Baldassarri, On Algebraic Solutions of Lamé's Differential Equation, J. Differential Equations, 41:44-58, 1981. MR0626620(84a:34006)

[BD] F. Baldassarri and B. Dwork, On Second Order Differential Equations with Algebraic Solutions, Amer. J. Math., 101:42-76, 1979. MR0527825 (81d:34002)

[BW] Frits Beukers and Alexa van der Waall, Lamé Equations with Algebraic Solutions, J. Differential Equations, 197:1-25, 2004. MR2030146 (2004j:34202)

[Chi] F. Chiarellotto, On Lamé Operators which are Pull-Backs of Hypergeometric Ones, Trans. Amer. Math. Soc., 347(8):2753-2780, 1995. MR1308004 (96e:34008)

[Cop] F. Coppi, Tesi di Laurea, Universitá di Padova, 1992.

[Hec] E. Hecke, Zur Theorie der Elliptischen Modulfunktionen, Math. Ann., 97:210-242, 1926. MR 1512360

[Lit1] R. Litcanu, Counting Lamé Differential Operators, Rend. Sem. Mat. Univ. Padova, 107:191-208, 2002. MR:1926211 (2003h:34186)

[Lit2] L Lamé Operators with Finite Monodromy, to appear.

[LZ] S. G. Lando and A. K. Zvonkin, Graphs on Surfaces and their Applications, Encyclopaedia of Mathematical Sciences, Vol. 141, Springer, Berlin, 2004. MR2036721 (2005b:14068)

[Poo] E. G. C. Poole, Introduction to the Theory of Linear Differential Equations, Oxford Univ. Press, London, 1936. MR0111886 (22:2746)

[vdW] Alexa van der Waall, Lamé Equations with Finite Monodromy, Universiteit Utrecht, Utrecht, 2002, Thesis. On-line reference: http://www.library.uu.nl/digiarchief/dip/diss/ 2002-0530-113355/inhoud.htm.

[WW] E. T. Whittaker and G. N. Watson, A Course of Modern Analysis, Cambridge Univ. Press, Cambridge, 1950, Reprint of the 1927 fourth edition. MR1424469 (97k:01072)

Department of Mathematics, Utrecht University, Budapestlaan 6, 3584 CD Utrecht, The Netherlands

E-mail address: dahmen@math.uu.nl 